\title{
HBOC in Europe
}

\author{
Hanne Meijers-Heijboer* \\ Department of Clinical Genetics, Academisch Medisch Centrum - Universiteit \\ van Amsterdam (AMC-UvA) and Department of Clinical Genetics, VU University \\ Medical Center Amsterdam, The Netherlands
}

DOI: http://dx.doi.org/10.19106/JMedScieSup0048042016011

\begin{abstract}
Europe has contributed to the majority of high-impact papers on genes and risk alleles in breast cancer and ovarian cancer susceptibility. Consortia like the Breast Cancer Linkage Consortium, Breast Cancer Association Consortium, and the Consortium of Investigators on Modifying genes in $B R C A 1 / 2$, started from the nineties of the last century on. Many highly motivated participants throughout Europe, the US, Australia, and elsewhere contributed to the formation of huge data sets. Instrumental of the success of the consortia was also the leadership and knowledge of Dough Easton, UK, on the statistics of cancer genetics. The consortia papers have produced the data from which national guidelines in HBOC were formulated in West-Europe, the US, and worldwide. Genetic testing for HBOC is accepted and funded in most Western-European countries. Two decades after the identification of $B R C A 1$ and $B R C A 2$, the attitudes towards genetic testing for $\mathrm{HBOC}$ are changing and becoming more 'common' practice. This offers opportunities to organize cancer genetics care more efficiently and at lower costs, while informed decision making and consent of the patients remain in place.
\end{abstract}

Keywords: BRCA 1, BRCA2, HBOC, Europe, Founder mutations, linkage

Corresponding author: H.Meijers-Heijboer@vumc.nl 